\title{
Ergreifen wir die Initiative für unsere Kinder und Jugendlichen!
}

\section{Carlos Beat Quinto}

Dr. med., Mitglied des FMH-Zentralvorstandes, Departementsverantwortlicher Public Health und Gesundheitsberufe

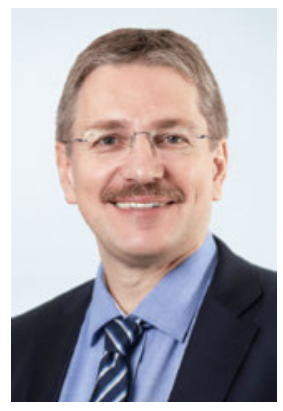

Ärztinnen und Ärzte sehen täglich die Folgen des Tabakkonsums in der Schweiz. Sie betreuen Patientinnen und Patienten mit Lungen-, Herz-, Gefäss-, Tumorund anderen tabakbedingten Krankheiten. Viele dieser Erkrankungen könnten vermieden werden, denn sie stehen in direktem Zusammenhang mit dem Tabakkonsum. Rauchende finden sich zudem überproportional in den niedrigsten sozioökonomischen Schichten. Rauchen ist somit auch eine Ursache erheblicher gesundheitlicher Ungleichheit.

Deshalb ist es unsere Aufgabe und unsere Pflicht, Kinder und Jugendliche vor dem Einstieg zu bewahren und uns für eine adäquate gesetzliche Regelung der Tabakprodukte einzusetzen. Waren Tabakprodukte bisher im Lebensmittelgesetz verankert, wurde im Rahmen der letzten Revision folgerichtig entschieden,

Mit einem umfassenden Werbeverbot wird der Tabakkonsum in Ländern mit hohem Einkommen um durchschnittlich 7 Prozent gesenkt.

die Tabakprodukte zu entfernen und in einem eigenen Gesetz zu regeln: dem Tabakproduktegesetz.

Da kein anderes Lebensmittel vergleichbare schädliche Auswirkungen auf die Konsumierenden hat, erhoffte sich die Ärzteschaft eine gesetzliche Regelung, welche den Mindeststandard der von der Schweiz 2004 unterschriebenen Tabak-Rahmenkonvention (FCTC) abdeckt. Doch dank Tabakindustrie-Lobbying hat die Schweiz als bald einziges Land auf dem Kontinent die FCTC noch immer nicht ratifiziert.

Schade, denn die neue Gesetzesgrundlage würde heute die Möglichkeit bieten, zu verhindern, dass Kinder und Jugendliche unsere Patienten von morgen werden. Gerade im Hinblick darauf, dass der Tabakkonsum nach wie vor zu den grössten Problemen der öffentlichen Gesundheit gehört und Folgekosten in Milliardenhöhe für Prämien- und Steuerzahler verursacht - und Gewinne in gleicher Höhe für die Tabakindustrie -, erscheint der überarbeitete Gesetzesentwurf mut- und weitgehend wirkungslos.

Eine wirksame Prävention muss ein wirksames, zeitgemässes Werbeverbot beinhalten. Denn die Tabak- industrie weiss Werbung als subtiles Instrument für sich zu nutzen. Ihre Werbung ist omnipräsent, und dies ohne dass es gross auffällt. Musikfestivals, Kino, Bars, Verkaufsstellen und private Anlässe sind nur eine Auswahl beliebter Orte, um den Glimmstängel und das damit angeblich verbundene Freiheitsgefühl zu verherrlichen.

Gerade Kinder und Jugendliche reagieren sensibel auf Werbung. Es erstaunt daher nicht, dass empirische Beobachtungen zeigen, dass die Tabakindustrie ihre Strategien besonders auf Jugendliche ausrichtet. Werbeorte, Sprache und Gestaltung zielen darauf $a b$, Jugendliche anzusprechen. Jedes Mittel ist erlaubt, um Tabak bei Kindern und Jugendlichen bekannt zu machen. Dies steht in komplettem Widerspruch zum gesetzlichen Verkaufsverbot.

In der Schweiz sterben jedes Jahr rund 9500 Menschen an den Folgen des Rauchens. Untersuchungen zeigen auch, dass 57 Prozent der Rauchenden bereits als Minderjährige mit dem Tabakkonsum beginnen. Und wissenschaftliche Studien belegen, dass Jugendliche, die häufig mit Tabakwerbung in Kontakt kommen, eher zu Rauchern werden.

Die FMH unterstützt deshalb die Initiative «Ja zum Schutz der Kinder und Jugendlichen vor Tabakwerbung». Ziel der Initiative ist es, Werbung für Tabakprodukte, die Kinder oder Jugendliche erreicht, einzu-

\section{Wo ein Kaufverbot gilt, soll auch nicht dafür geworben werden dürfen.}

dämmen. Konkret soll Werbung auf Plakaten im öffentlichen Raum in allen Schweizer Kantonen verboten werden. Auch Kinowerbung, Inserate, FestivalSponsoring und Onlinewerbung für Tabak sollen in Zukunft nicht mehr erlaubt sein.

Die Initiative ist liberal im wahren Sinn: Sie schafft Freiheit von Abhängigkeit und schränkt den erwachsenen Bürger und seine Selbstverantwortung in keiner Weise ein.

Es liegt in unserer Verantwortung, dass die Kinder und Jugendlichen von heute nicht unsere Patienten von morgen werden. Unterzeichnen Sie jetzt! 